\title{
Le Radeau des Cimes au Parc national de Masoala (Madagascar). Deuxième partie : recherches entomolo- giques dans la canopée supérieure et le sous-bois de la forêt humide
}

\author{
Olga Ravoahangimalala Ramilijaona ${ }^{1}$, Henri-Pierre Aberlenc ${ }^{2}$, \\ Héctor Barrios ${ }^{3}$, Landinirina Andriamanantenina, Gianfranco \\ Curletti $^{4}$, Joël Minet ${ }^{5}$, Laurence Beaudoin-Ollivier ${ }^{6}$, Hantanirina Liss \\ Rakotoarivony ${ }^{1}$ et Désiré Randriamasimanana1
}

1 Département de biologie anomale, Facu'té des sciences, Université d'Antananarivo, B.P. 906, Antananarivo 101, Madagascar - oramilij@yahoo.fr

2 Cirad Bios, TA A-55/L, UMR 55 (CBGP), Campus international de Baillarguet (CSIRO), F-34398 Montpellier Cedex 5 - henri-pierre.aberlenc@cirad.fr

3 Programa de Maestría en Entomología, Universidad de Panamá, Panamá City, Panamá. hbarrios@ancon.up.ac.pa

4 Museo Civico di Storia Naturale, Cas. Post. 89, I-10022 Carmagnola (TO) - giancurletti@tiscali.it

5 Muséum national d'histoire naturelle, Département systématique \& évolution, USM 602, Case Postale n 50 (Entomologie), F-75231 Paris Cedex 05 - minet@mnhn.fr

6 Cirad Bios, UPR 31, TA A-31/02, F-34398 Montpellier Cedex 5 - laurence.ollivier@cirad.fr

Résumé. - Notre étude porte sur l'analyse quantitative de la stratification verticale (sous-bois et canopée supérieure) de l'entomofaune de neuf sites en forêt tropicale humide de la presqu'île de Masoala, sur le rivage oriental de la baie d'Antongil (Madagascar). Nous avons pu accéder à

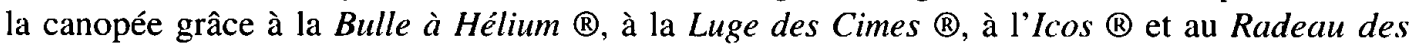
Cimes $\left({ }^{B}\right.$. Les Insectes ont été collectés par battage du feuillage et avec des pièges jaunes à glu. Les Arthropodes de la canopée supérieure sont significativement plus abondants que dans le sous-bois. Ce type de stratification verticale de l'entomofaune de la forêt tropicale confirme dans ses grandes lignes, malgré quelques différences, le modèle que nous avions établi au Cameroun et affiné au Gabon. L'analyse par guildes montre que certains de ces groupes ont nettement une strate préférentielle (en particulier les Phyllophages et les Piqueurs-suceurs pour la canopée), ce qui est directement fonction de la répartition verticale des ressources alimentaires. L'assignation à une guilde semble être un instrument pertinent pour étudier l'écologie et la stratification verticale des Insectes de la forêt tropicale.

Mots-clés. - Arthropoda, Insecta, biodiversité, stratification verticale, guildes, phyllophages, piqueurs-suceurs, baie d'Antongil, battage, pièges jaunes à glu, Radeau des Cimes, Luge des Cimes, Bulle à Hélium.

The Canopy Raft in the Masoala National Park (Madagascar). 2. Entomological research in the upper canopy and understorey of the rainforest

Summary. - Our study deals with the quantitative analysis of the vertical stratification (understorey and canopy) of the entomofauna of nine field sites in tropical rainforests of the Masoala peninsula, east of the Baie d'Antongil (Madagascar). We reached the canopy using the "Bulle à Hélium" (helium balloon) $®$, the "Icos", the "Luge des Cimes" (canopy sledge) $®$, and the "Radeau des Cimes" (canopy raft) ${ }^{\circledR}$. Insects were collected by leaf beating and with yellow sticky traps. Arthropods from the canopy are significantly more abondant than in understory.

Accepté pour publication le 21 juin 2007. 
This vertical stratification of a tropical forest confirms generally, despite a few differences, a model specified in Cameroon, and improved in Gabon. The guild analysis shows than some of these groups have a preferred stratum (in particular leaf-feeders and sap-suckers for the canopy). This is correlated with the vertical distribution of food resources. The guild assignment is a pertinent way to study ecology and vertical stratification of insects in tropical rainforests.

Keywords. - Arthropoda, Insecta, biodiversity, vertical stratification, guilds, leaf-feeders, sap-suckers, baie d'Antongil, beating, yellow sticky traps, canopy raft, canopy sledge, helium balloon.

\section{INTRODUCTION}

L'un des plus graves reculs de la biodiversité mondiale de l'histoire de notre planète est en cours. Résultant notamment de la pollution et de la disparition des habitats (WILSON, 1988, 1989, 1992), il se manifeste par la forte diminution des effectifs des populations qui va jusqu'à l'extinction de nombreuses espèces végétales et animales (ERWIN, 1991, 1995). Les systématiciens ont nommé et décrit près de 900000 espèces d'Insectes (MARTINEZ \& GAUVRIT, 1997), dont plus de la moitié vivent dans les forêts tropicales. Il faut également souligner que les Insectes représentent $70 \%$ de la biodiversité totale des Arthropodes (WILSON, 1988). Toutefois, l'estimation du nombre total d'espèces d'Insectes suscite un intense débat depuis de nombreuses années (BASSET $e t$ al., 1996). Par ailleurs, nos connaissances sur l'écologie des Insectes tropicaux restent très insuffisantes (JANZEN, 1988 ; BASSET, 1992 ; BASSET \& NOVOTNY, 1999 ; BARRIOS, 2003).

Dans les forêts tropicales, la production primaire et la biodiversité semblent être réparties au hasard. Les conditions biotiques varient radicalement selon une stratification verticale de la forêt. La canopée supérieure est directement exposée au rayonnement solaire. Les températures à midi sont de 5 à $8^{\circ}$ degrés plus élevées et l'humidité relative est inférieure d'environ $30 \%$ à celle du sous-bois $30 \mathrm{~m}$ plus bas, à l'ombre (WRIGHT \& COLLEY, 1994 ; BASSET et al., 2001). Les fluctuations du vent, de l'humidité relative et la condensation de l'eau la nuit sont nettement plus élevées dans la canopée que dans le sous-bois (BLANC, 1990 ; PARKER, 1995). Comme l'a montré Fowler (1985), la plupart des arbres tropicaux adultes sont 100 fois plus hauts que leurs plantules, alors que ce rapport de hauteur est de 30 dans les régions dites tempérées. Le feuillage de ces arbres tropicaux peut présenter de nombreux microclimats différents de ceux du sous-bois, ce qui pourrait fortement influencer les stratégies de recherche de nourriture végétale par les Insectes (SHELLY, 1985 ; RouBIK, 1993). La densité de surface foliaire et l'abondance des jeunes feuilles, fleurs et graines est plus élevée dans la canopée supérieure que dans le sous-bois (PARKER, 1995 ; HALLÉ, 1998). Les boutons floraux sont très bien protégés dans la canopée supérieure pour éviter la déshydratation et résister aux attaques des phytophages (BELL et al., 1999). Globalement, tous ces facteurs varient beaucoup du sous-bois à la canopée supérieure, cette variation ayant une répercussion sur la répartition des Insectes phytophages (SCHOWALTER et al., 1986).

En réalité, pour résumer, quatre catégories de facteurs affectent la répartition verticale des espèces d'Arthropodes en forêt tropicale (BASSET et al., 2003) : 
1 - les facteurs abiotiques;

2 - la physionomie de la forêt;

3 - la disponibilité des ressources ;

4 - le comportement des Arthropodes.

Cela suggère que les espèces qui y vivent sont spécialisées et se sont adaptées aux conditions microclimatiques prévalant dans la canopée supérieure (où les variations sont extrêmes) et dans le sous-bois (où elles sont davantage tamponnées).

Nous poursuivons la publication des résultats que notre équipe entomologique a obtenus au cours de la mission du Radeau des Cimes à Madagascar en octobre et novembre 2001 (VITALI, 2006; ABERLENC et al., 2007). Il s'agissait de collecter des macroarthropodes adultes, en particulier des Insectes phytophages.

Les données que nous avons recueillies à Masoala permettent de vérifier si les modèles élaborés au cours de nos recherches antérieures à Campo au Cameroun (BASSET et al., 1992) et à la Makandé au Gabon (BASSET et al., 2001) ont une portée générale.

\section{MATÉRIEL ET MÉTHODES}

\section{Zone d'étude et accès à la canopée}

Les Arthropodes ont été collectés dans la forêt tropicale de basse altitude du Parc national de Masoala (Madagascar), à la pointe Tampolo, sur la baie d'Antongil. La moyenne annuelle des précipitations est de $548 \mathrm{~mm}$ et la température moyenne annuelle est de $23,4^{\circ}$ (1993, données WCS [Wildlife Conservation Society]). La hauteur de la canopée, assez faible, varie de 20 à $35 \mathrm{~m}$. Les caractéristiques générales de la végétation ont été décrites par PAULIAN (1961). La saison (octobre-novembre 2001) n'était pas optimale pour la collecte des Insectes dans cette région du monde.

Au cours de la mission, notre équipe a travaillé dans neuf stations distantes de 1,5 à $5 \mathrm{~km}$, qui ont été numérotées de 1 à 9 (voir tableau I). Nous avons pu accéder à la canopée grâce à l'aide logistique de l'équipe d'Océan vert. Nous avons pu utiliser le Radeau des Cimes (Ebersolt, 1990, 1999) (photos 3 et 4), la Luge des Cimes (Ebersolt, 1990) (photos 6 et 7), la Bulle des Cimes (Cleyet-Marrel, 1999) (photo 1) et l'Icos II (photo 10). Nous avons prélevé des échantillons d'Arthropodes dans le feuillage de la canopée supérieure accessible à la périphérie du Radeau et de l'Icos II, le long du transect de la Bulle (photo 2) et au cours des vols de la Luge (photo 9). Le périmètre du Radeau est d'environ $80 \mathrm{~m}$. Nous avons utilisé le transect de la Bulle sur une longueur d'environ $200 \mathrm{~m}$. Nous avons aussi collecté des échantillons dans la canopée supérieure à l'aide de la Luge en divers sites autour du camp de base, au cours de vols accomplis tôt le matin (photos 6 à 9). Pour presque chaque prélèvement dans la canopée supérieure, nous avons pu prélever des échantillons dans la zone correspondante du sous-bois. 
Deux types de forêts étaient accessibles depuis le camp (photos 1 et 8) :

1 - Près de la côte, une forêt humide de type littoral en grande partie secondarisée (avec quelques lambeaux de la forêt primaire qu'on retrouve à plus haute altitude) sur des reliefs faibles et d'altitude très faible (entre zéro et quelques dizaines de mètres). On découvre en la survolant des clairières ouvertes de main d'homme. Le camp de base était installé à l'emplacement d'une ancienne usine abandonnée équipée d'un embarcadère sur la baie d'Antongil, qui était alimentée en troncs d'arbres par une ligne étroite de chemin de fer longeant la côte, vers le sud (seules demeurent les fondations des installations ; les rails ont disparu, ne subsistent que quelques traverses et la tranchée de la voie) [accès à la canopée : Bulle et Luge].

2 - Plus loin de la côte, une forêt primaire (ou secondaire très ancienne) sur les contreforts très pentus du massif montagneux (culminant vers $700 \mathrm{~m}$ ), partiellement secondarisée, car elle aussi est trouée de clairières pour des cultures [accès à la canopée : Icos II et Radeau].

Dans ces deux types de forêts, on peut distinguer facilement la canopée supérieure et le sous-bois.

\section{Méthodes d'échantillonnage}

Nos échantillons ont été prélevés dans chaque station afin d'obtenir :

a) la densité d'Arthropodes capturés par les pièges à glu jaunes ;

b) la densité d'Arthropodes par surface de feuillage au battage.

Nous avons placé dans les stations d'échantillonnage 65 pièges à glu jaunes au niveau de la canopée supérieure et 76 dans le sous-bois. Les pièges jaunes mesuraient $13,3 \times 24 \mathrm{~cm}$ et, comme ils étaient englués des deux côtés, la surface totale de collecte était de $638,4 \mathrm{~cm}^{2}$ par piège. Dans la canopée supérieure, les pièges furent placés sur le feuillage à la périphérie du Radeau (photos 3 et 4), au niveau du transect de la Bulle (photos 1 et 2) ou dans la végétation directement accessible autour de l'Icos II (photo 10). Dans le sous-bois, les pièges jaunes ont été placés à une hauteur d'environ $1,5 \mathrm{~m}$ le long de transects de quelques dizaines de mètres situés dans une zone aussi proche que possible de la verticale du Radeau ou de l'Icos II, ou le long du layon ouvert au sol sous la corde-guide de la Bulle. Dans chaque station de prélèvement, pour chaque strate, les pièges jaunes ont été exposés en principe pendant quatre jours. Les Insectes englués étaient pour la plupart irrécupérables. Ils furent triés en partie au laboratoire du camp de base et en partie au CIRAD (Montpellier) et à l'Université de Panama City (Universidad de Panamá).

Les Arthropodes ont été recueillis au battage avec un battoir en tissu blanc tendu sur deux bâtons en X (instrument à base carrée, de forme subpyramidale [pointe en bas], d'environ $0,397 \mathrm{~m}^{2}$, avec les côtés inclinés à environ $45^{\circ}$, terminés par une ouverture circulaire de $7 \mathrm{~cm}$ de diamètre à l'extrémité de laquelle on attache, avec un élastique, une poche en plastique amovible : photo 9). Cet outil est placé sous le feuillage, en veillant à ce qu'une couche de feuilles recouvre approximativement toute la surface col- 
lectrice. On fait tomber les Arthropodes par trois vigoureux coups de bâton dans le feuillage, puis on les balaye vers la poche collectrice en plastique par de délicats coups de pinceau. La poche en plastique est ensuite remplacée par une nouvelle poche. Le contenu des poches a été trié au laboratoire du camp de base (photo 5) et les échantillons ont été conservés en éthanol à $70^{\circ}$. Nous avons recueilli au total 234 poches dans la canopée supérieure et 212 dans le sous-bois. Dans la canopée supérieure, les échantillons ont été recueillis à la périphérie du Radeau ou avec la Luge. Au niveau du sousbois, les échantillons ont été collectés à une hauteur inférieure à $2 \mathrm{~m}$, soit dans un secteur le plus proche possible de la verticale du Radeau, soit (pour pouvoir comparer avec les échantillons obtenus avec la Luge) le long du layon ouvert sous la corde directrice de la Bulle ou en divers points de la forêt selon un transect d'approximativement $200 \mathrm{~m}$. Nous n'avons pas fait de battage dans la canopée supérieure autour de l'Icos II, car la périphérie accessible était trop petite, ni avec la Bulle à cause de sa grande instabilité et du vent très capricieux. Un biais dû à la différence de densité foliaire entre la canopée et le sous-bois ne peut être exclu.

\section{Traitement des échantillons}

Les Arthropodes furent comptés et identifiés au niveau de la famille et dans certains cas à des niveaux taxinomiques supérieurs. Les Insectes phytophages collectés au battage ont été triés jusqu'au niveau de la morpho-espèce. Les échantillons englués sur les pièges jaunes étaient abîmés, ce qui a entraîné des difficultés pour les identifier correctement, c'est pourquoi on s'est contenté d'aller seulement au niveau de la famille. Chaque échantillon a été classé dans une guilde selon la classification des Arthropodes arboricoles proposée par MORAN \& SOUTHWOOD (1982) :

$\begin{array}{ll}\text { - Phyllophages (Phy) } & \text { [leaf-chewers] } \\ \text { - Piqueurs-suceurs de sève (Ps) } & \text { [sap-suckers] } \\ \text { - Mycophages (Myc) } & \text { [fungal feeders] } \\ \text { - Prédateurs d'Insectes (Pi) } & \text { [insect predators] } \\ \text { - Autres prédateurs (Ap) } & \text { [other predators] } \\ \text { - Parasitoïdes (Par) } & \text { [parasitoids] } \\ \text { - Xylophages (Xyl) } & \text { [wood-eaters] } \\ \text { - Saprophages (Sap) } & \text { [scavenger] } \\ \text { - Fourmis (For) } & \text { [ants] } \\ \text { - Touristes (Tou) } & \text { [tourists] } \\ \text { - Inconnus (Inc) } & \text { [unknown] }\end{array}$

Nous avons inclus les groupes Phy et Ps dans les Phytophages (auxquels, en théorie, devraient aussi se rattacher les Xylophages). Les « Touristes » sont les Insectes trouvés sur des arbres que nous estimons être utilisés comme des refuges ou pour diverses activités, mais pas pour s'y alimenter. 


\section{Analyses statistiques}

Nous avons utilisé les logiciels Biota (CoLwell, 1997), Microsoft Excel et Systat 7 pour traiter les données. D'une manière générale, les données recueillies n'ont pas une distribution normale, car l'abondance des Arthropodes varie beaucoup d'un échantillon à l'autre. Pour cette raison, nous présentons les données sous forme de moyennes ou nous mentionnons le nombre total d'individus collectés. L'influence des sites de collecte sur la densité des échantillons prélevés a été analysée avec le test de Kruskal-Wallis et Friedman et l'influence de la strate avec le test de Mann-Whitney. Nous avons fait des analyses générales de l'ensemble des données, puis nous avons traité à part le matériel prélevé dans les stations de collecte $3,4,5,6$ et 8 pour affiner l'analyse. Ces stations de collecte correspondent en effet à un effort d'échantillonnage plus homogène (tableau I), alors que dans les stations de collecte 1, 2, 7 et 9 , nous avons recueilli des échantillons seulement dans la canopée ou seulement dans le sous-bois ou dans les deux strates mais avec peu d'échantillons, ce qui nuit à l'analyse.

\section{RÉSULTATS}

\section{Généralités sur la composition faunistique}

Au cours de cette étude :

- nous avons collecté au total 11219 Arthropodes au moyen de 587 prélèvements effectués dans les deux strates (canopée supérieure et sous-bois) ;

- nous avons posé 141 pièges jauncs ( 65 dans la canopée supérieure et 76 dans le sous-bois) qui ont permis de collecter 8008 Arthropodes (3 954 dans la canopée supérieure et 4054 dans le sous-bois) ;

- nous avons recueilli 446 échantillons de battage (234 dans la canopée supérieure et 212 dans le sous-bois) qui ont permis de collecter 3211 Arthropodes (1 647 dans la canopée supérieure et 1564 dans le sous-bois).

Les Arthropodes collectés se répartissent entre 14 ordres d'Insectes $(92,4 \%)$ et les Araignées (7,6\%). Nous avons trouvé 5601 (49,92\%) exemplaires d'Arthropodes dans la canopée supérieure et $5618(50,08 \%)$ exemplaires d'Arthropodes dans le sous-bois (tableau II). Les Coléoptères, Diptères, Hémiptères, Hyménoptères et Araignées représentent $85,43 \%$ du total.

Sur un total de 122 taxa différents collectés, 109 l'ont été dans la canopée supérieure et 125 dans le sous-bois. Certains Arthropodes n'ont pu être identifiés au niveau de la famille, ceci en raison d'une dégradation plus ou moins importante (spécialement dans le cas des pièges jaunes) : il s'agit notamment d'une partie des Diptères et des Araignées.

Parmi les échantillons prélevés se détachent quelques groupes clairement prédominants :

1 - Phyllophages (Phy) [Chrysomelidae, Curculionidae et Tettigonidae] ;

2 - Piqueurs-suceurs (Ps) [Aphididae, Cercopidae et Cicadellidae] ;

3 - Prédateurs d'Insectes (Pi) [Cleridae et Coccinellidae] ; 


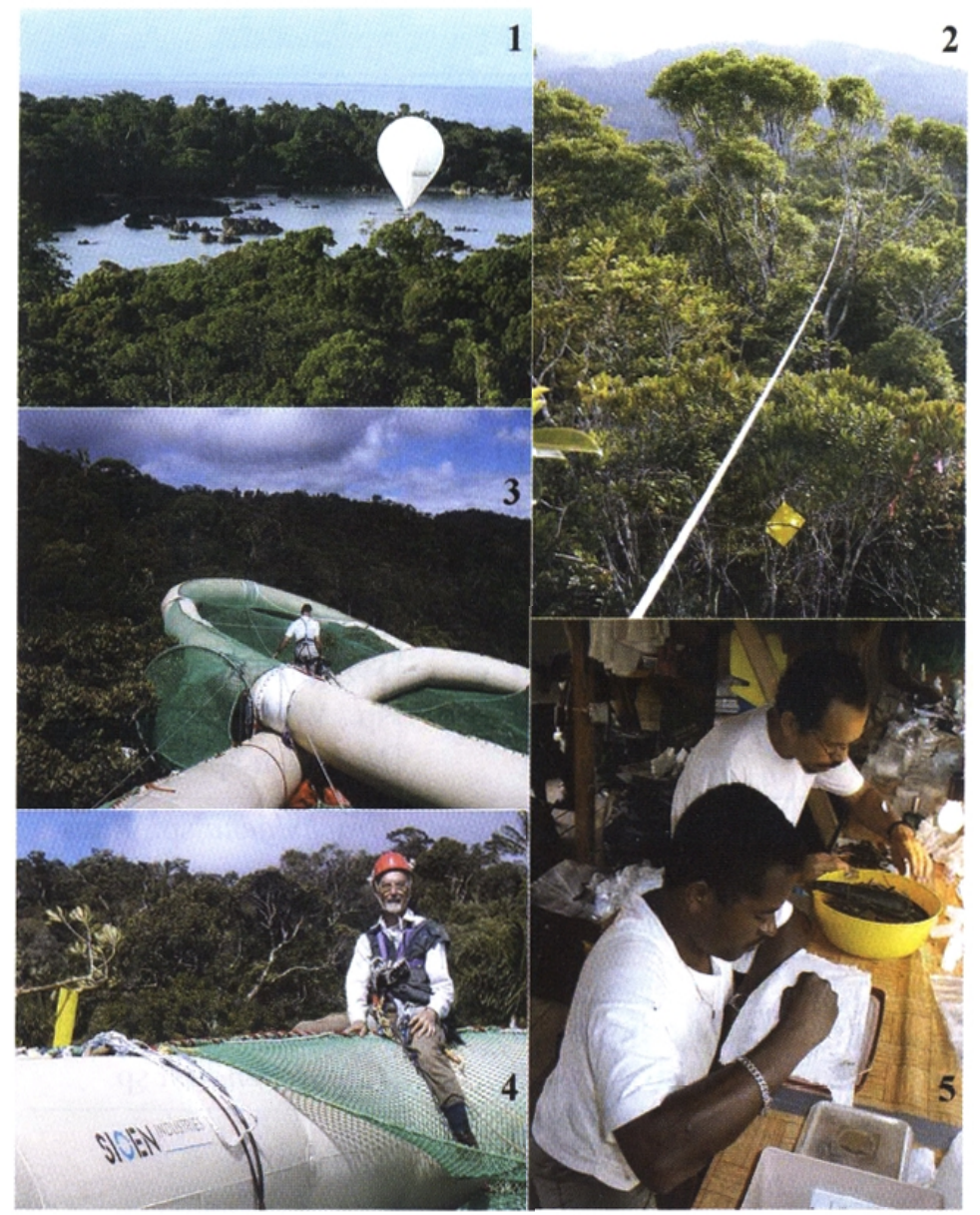

1 - La Bulle à Hélium sur la canopée de la forêt littorale [photo HS].

2 - Un piège à glu jaune dans la canopée, près de la cordeguide de la Bulle à Hélium [photo HPA].

3 - Le Radeau des Cimes sur la canopée [photo HPA].

4 - Gianfranco Curletti sur le Radeau des Cimes, à côté du piège à glu jaune qu'il vient de poser dans la canopée [photo HPA].

5 - Héctor Barrios et Désiré Randriamasimanana au laboratoire du camp de base, triant les échantillons biotés au battage [photo HPA].

6 - Le dirigeable et la luge en vol au-dessus de la canopée [photo HPA].

7 - La luge vue depuis le dirigeable : François Klene accompagne Héctor Barrios et Désiré Randriamasimanana qui ont chacun un battoir [photo HS].

8 - La canopée vue depuis la luge en vol. On voit nettement la différence entre la forêt littorale (avec une clairière d'origine anthropique au premier plan à droite) et la forêt de pente [photo HPA].

9 - Héctor Barrios dans la luge en vol montant une poche en plastique sous son battoir [photo HS].

10 - L'Icos II vu depuis le sous-bois, avec divers pièges suspendus [photo HPA].
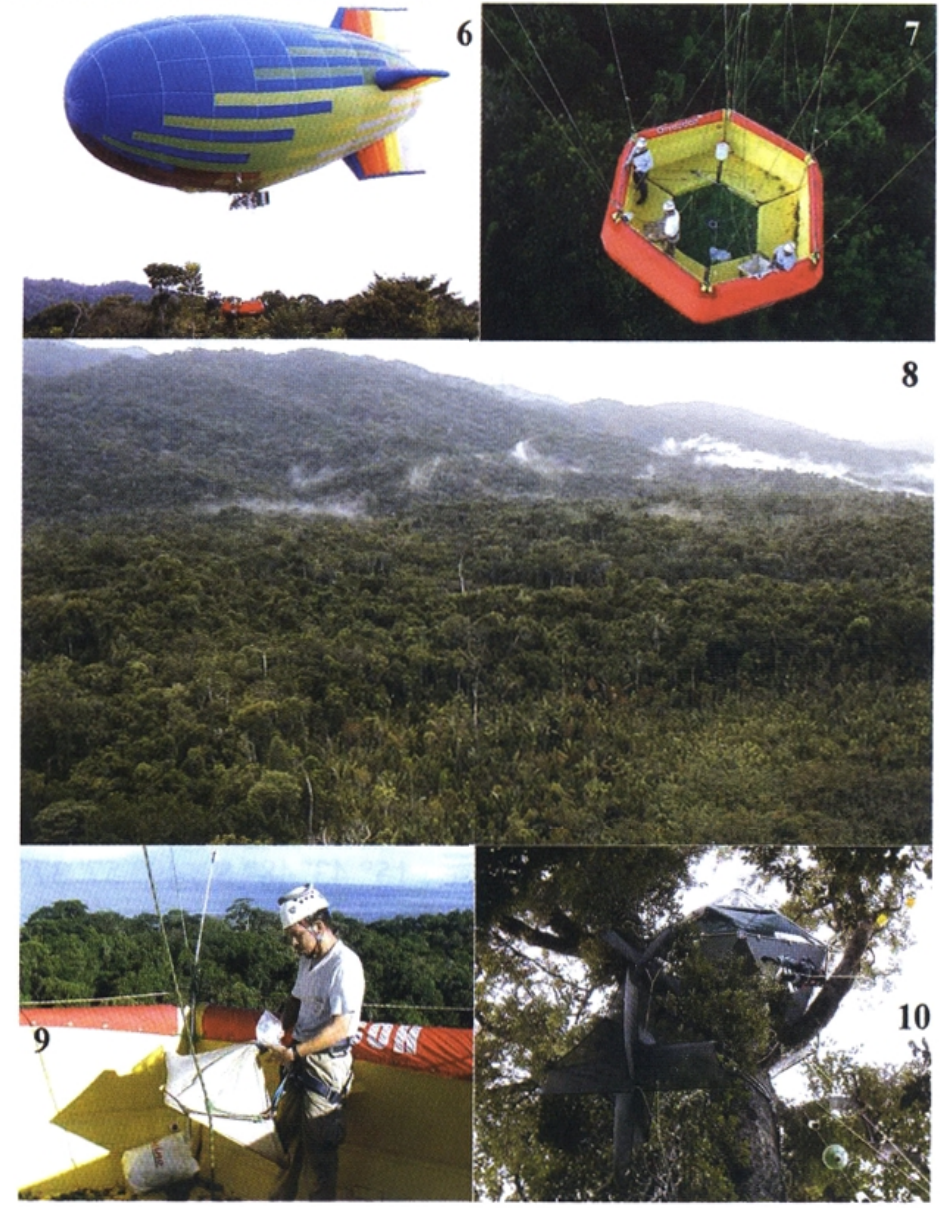


\begin{tabular}{|c|c|c|c|c|c|}
\hline $\begin{array}{l}\text { Accès à la } \\
\text { canopée }\end{array}$ & $\begin{array}{l}\text { Hauteur } \\
\text { de la } \\
\text { canopée } \\
\text { (en m) }\end{array}$ & $\begin{array}{c}\text { Dates des } \\
\text { prélèvements }\end{array}$ & Battage & $\begin{array}{l}\text { Pièges à } \\
\text { glu jaunes }\end{array}$ & $\begin{array}{c}\text { Principaux arbres } \\
\text { émergents }\end{array}$ \\
\hline $\begin{array}{l}\text { Bulle } \\
\text { (transect) }\end{array}$ & 25 & $\begin{array}{l}\text { du } 07 \text { au } \\
\text { 17.XI.2001 }\end{array}$ & $\begin{array}{l}\mathrm{n}=0 \mathrm{can} \\
\mathrm{n}=21 \mathrm{sb}\end{array}$ & $\begin{array}{l}\mathrm{n}=6 \mathrm{can} \\
\mathrm{n}=10 \mathrm{sb}\end{array}$ & 13 familles \\
\hline Icos II & 35 & $\begin{array}{l}\text { du } 28 . X \text { au } \\
17 . X I .2001\end{array}$ & $\begin{array}{l}\mathrm{n}=0 \text { can } \\
\mathrm{n}=0 \mathrm{sb}\end{array}$ & $\begin{array}{l}\mathrm{n}=20 \mathrm{can} \\
\mathrm{n}=10 \mathrm{sb}\end{array}$ & $\begin{array}{l}\text { Xylopia } \mathrm{sp.} \\
\text { Magnistipula sp. }\end{array}$ \\
\hline $\begin{array}{l}\text { Radeau } \\
\text { (site unique) }\end{array}$ & $30-35$ & $\begin{array}{l}\text { du ler au } \\
\text { 17.XI.2001 }\end{array}$ & $\begin{array}{l}\mathrm{n}=57 \mathrm{can} \\
\mathrm{n}=57 \mathrm{sb}\end{array}$ & $\begin{array}{l}\mathrm{n}=39 \mathrm{can} \\
\mathrm{n}=40 \mathrm{sb}\end{array}$ & 9 familles \\
\hline $\begin{array}{l}\text { Luge } \\
(\text { vol 1) }\end{array}$ & $30-35$ & 02.XI.2001 & $\begin{array}{l}\mathrm{n}=31 \mathrm{can} \\
\mathrm{n}=45 \mathrm{sb}\end{array}$ & $\begin{array}{l}\mathrm{n}=0 \\
\mathrm{n}=0\end{array}$ & $\begin{array}{l}\text { Phyllarthron sp., } \\
\text { Syzygium sp., Hirtella } \\
\text { sp., Weinmannia } \text { sp., } \\
\text { Noronhia } \text { sp. }\end{array}$ \\
\hline $\begin{array}{l}\text { Luge } \\
(\text { vol 2) }\end{array}$ & $30-35$ & 03.XI.2001 & $\begin{array}{l}\mathrm{n}=45 \mathrm{can} \\
\mathrm{n}=48 \mathrm{sb}\end{array}$ & $\begin{array}{l}\mathrm{n}=0 \\
\mathrm{n}=0\end{array}$ & $\begin{array}{l}\text { Brachylaena sp., Rhus } \\
\text { sp., Uapaca } \mathrm{sp} . \\
\text { Noronhia } \mathrm{sp} ., \text { Norhoria } \\
\text { sp., Breonia } \mathrm{sp} .\end{array}$ \\
\hline $\begin{array}{l}\text { Luge } \\
(\text { vol 3) }\end{array}$ & $30-35$ & 12.XI.2001 & $\begin{array}{l}\mathrm{n}=21 \mathrm{can} \\
\mathrm{n}=20 \mathrm{sb}\end{array}$ & $\begin{array}{l}\mathrm{n}=0 \\
\mathrm{n}=0\end{array}$ & $\begin{array}{l}\text { Symphonia } \mathrm{sp} ., \\
\text { Capurodendron sp. }\end{array}$ \\
\hline $\begin{array}{l}\text { Luge } \\
\text { (vol 4) }\end{array}$ & $30-35$ & 15.XI.2001 & $\begin{array}{l}\mathrm{n}=30 \mathrm{can} \\
\mathrm{n}=0 \mathrm{sb}\end{array}$ & $\begin{array}{l}\mathrm{n}=0 \\
\mathrm{n}=0\end{array}$ & 10 familles \\
\hline $\begin{array}{l}\text { Luge } \\
\text { (vol 5) }\end{array}$ & $30-35$ & 16.XI.2001 & $\begin{array}{l}\mathrm{n}=50 \mathrm{can} \\
\mathrm{n}=21 \mathrm{sb}\end{array}$ & $\begin{array}{l}\mathrm{n}=0 \\
\mathrm{n}=0\end{array}$ & 16 familles \\
\hline $\begin{array}{l}\text { Aucun : forêt } \\
\text { près du fleuve } \\
\text { Tampolo }\end{array}$ & $30-35$ & $\begin{array}{l}\text { du 27.X au } \\
\text { 17.XI.2001 }\end{array}$ & $\begin{array}{l}\mathrm{n}=0 \mathrm{can} \\
\mathrm{n}=0 \mathrm{sb}\end{array}$ & $\begin{array}{l}\mathrm{n}=0 \\
\mathrm{n}=16 \mathrm{sb}\end{array}$ & 11 familles \\
\hline
\end{tabular}

Tableau I - Sites où des Arthropodes ont été collectés en forêt à Masoala (can = canopée, $\mathrm{sb}=$ sous-bois)

Les sites 2 (Icos II) et 3 (Radeau des Cimes) sont en forêt de pente de moyenne altitude. Tous les autres sites sont en forêt littorale à relief faiblement ondulé, très peu au-dessus du niveau de la mer, non loin du camp de base, pointe Tampolo, à l'embouchure du fleuve Tampolo.

Seuls les vols de luge relatifs à nos activités entomologiques sont numérotés (d'autres vols furent consacrés à d'autres programmes).

Coordonnées GPS $I \cos I I: 15^{\circ} 42^{\prime} 35,4^{\prime \prime} \mathrm{S} ; 4^{\circ} 58^{\prime} 13,2^{\prime \prime} \mathrm{E}$.

Coordonnées GPS Radeau : $15^{\circ} 42^{\prime} 77^{\prime \prime} \mathrm{S} ; 4^{\circ} 58^{\prime} 25^{\prime \prime} \mathrm{E}$.

Coordonnées GPS camp de base : $15^{\circ} 43^{\prime} 48^{\prime \prime} \mathrm{S} ; 49^{\circ} 57^{\prime} 37^{\prime \prime} \mathrm{E}$. 
Plantes communes sur les sites de collecte :

Anacardiaceae : Gluta tourtour, Rhus sp.

Annonaceae : Xylopia sp.

Asteraceae : Brachylaena sp.

Bignoniaceae : Phyllarthron sp.

Chrysobalanaceae : Hirtella sp., Magnistipula

sp.

Clusiaceae : Calophyllum sp., Symphonia sp.

Convolvulaceae : Merremia peltata

Cunoniaceae : Weinmannia sp.

Dilleniaceae

Euphorbiaceae : Euphorbia tetraptera, Uapaca

sp.

Fabaceae
Lauraceae

Malvaceae (= Sterculiaceae)

Melastomataceae : Dionycha sp.

Meliaceae

Moraceae

Myrsinaceae

Myrtaceae : notamment Syzygium emirnense

Oleaceae : Noronhia sp.

Rubiaceae : Breonia sp., Mussaenda sp.

Salicaceae (= Flacourtiaceae)

Sapotaceae : notamment Capurodendron sp.

Sphaerosepalaceae (= Rhopalocarpaceae)

\begin{tabular}{|l|c|c|c|}
\hline \multicolumn{1}{|c|}{ Taxa } & Canopée & Sous-bois & Total \\
\hline Coleoptera & 925 & 1149 & 2074 \\
\hline Collembola & 2 & 15 & 17 \\
\hline Dermaptera & 1 & 4 & 5 \\
\hline Diptera & 1450 & 1256 & 2706 \\
\hline Heteroptera & 94 & 68 & 162 \\
\hline « Homoptera » & 1383 & 561 & 2861 \\
\hline Hymenoptera & 1281 & 1580 & 6 \\
\hline Isoptera & 0 & 6 & 1 \\
\hline Lepidoptera & 11 & 36 & 373 \\
\hline Neuroptera & 0 & 1 & 63 \\
\hline Orthoptera & 147 & 226 & 98 \\
\hline « Psocoptera » & 41 & 22 & 11 \\
\hline Thysanoptera & 26 & 72 & 851 \\
\hline « Thysanura » & 0 & 11 & 11219 \\
\hline Araneae & 240 & 611 & 407 \\
\hline Total & 5361 & 5007 & \\
\hline
\end{tabular}

Tableau II - Nombre total d'exemplaires d'Arthropodes collectés dans la canopée et le sous-bois au battage et avec les pièges jaunes. Les groupes paraphylétiques sont placés entre guillemets 


\begin{tabular}{|l|c|c|c|}
\hline \multirow{2}{*}{ Taxa / Groupes écologiques } & \multicolumn{2}{|c|}{ Moyenne du nombre d'exemplaires } & Mann-Whitney \\
\cline { 2 - 4 } & Sous-bois & Canopée & U P \\
\hline Phyllophages (Phy) & $1,992(2,395)$ & $2,764(4,55)$ & 0,010 \\
\hline Chrysomelidae & $2,624(3,096)$ & $3,786(5,03)$ & 0,019 \\
\hline Curculionidae & $1,630(1,346)$ & $2,020(4,51)$ & 0,588 \\
\hline Tettigoniidae (*) & $1,217(0,513)$ & $1,125(0,35)$ & 0,320 \\
\hline Piqueurs-suceurs (Ps) & $2,160(2,712)$ & $4,219(6,56)$ & 0,000 \\
\hline \multicolumn{1}{|c|}{ Aphididae } & $1,318(0,568)$ & $6,308(6,31)$ & 0,000 \\
\hline Cicadellidae & $2,351(2,126)$ & $5,319(8,35)$ & 0,000 \\
\hline Cercopidae & $3,210(4,349)$ & $1,083(0,89)$ & 0,000 \\
\hline Mycophages (Myc) & $1,469(1,140)$ & $1,861(2,167)$ & 0,107 \\
\hline \multicolumn{1}{|c|}{ Phlaeothripidae (Bactrothrips) } & $1,235(0,562)$ & $1,000(0,0)$ & 0,502 \\
\hline Prédateurs d'Insectes (Pi) & $1,339(1,092)$ & $1,397(1,068)$ & 0,630 \\
\hline Autres prédateurs (Ap) & $3,199(1,876)$ & $1,832(1,110)$ & 0,000 \\
\hline Parasitö̈des (Par) & $9,515(15,881)$ & $6,759(11,438)$ & 0,114 \\
\hline Gallicoles (Gal) & $7,026(6,757)$ & $4,319(9,168)$ & 0,001 \\
\hline Xylophages (Xyl) & $1,573(1,077)$ & $1,386(0,959)$ & 0,250 \\
\hline Saprophages (Sap) & $3,216(6,571)$ & $2,965(5,350)$ & 0,394 \\
\hline \multicolumn{1}{|c|}{ Gryllidae } & $1,333(0,564)$ & $1,125(0,38)$ & 0,037 \\
\hline Formicidae (For) & $2,740(3,684)$ & $4,277(8,285)$ & 0,069 \\
\hline Touristes (Tou) & $1,260(0,567)$ & $3,406(3,606)$ & 0,000 \\
\hline Inconnus (Inc) & $1,618(1,027)$ & $5,109(4,691)$ & 0,000 \\
\hline
\end{tabular}

Tableau III - Moyenne par groupe écologique du nombre d'exemplaires d'Arthropodes collectés au battage et avec les pièges jaunes dans la canopée et le sous-bois.

Comme dans les tableaux suivants, les probabilités significatives sont mises en italique et la déviation standard (DS) est indiquée entre parenthèses.

(*) Si certains Tettigoniidae sont phyllophages, d'autres sont prédateurs d'Insectes. Faute de pouvoir départager nos échantillons, nous les avons regroupés dans le seul groupe Phy.

\begin{tabular}{|c|c|c|c|}
\hline \multirow{2}{*}{$\mathbf{N}^{\circ}$} & \multicolumn{2}{|c|}{ Moyenne du nombre d'exemplaires } & Mann-Whitney P \\
\cline { 2 - 4 } & Sous-bois & Canopée & \\
\hline 1 & $3,091(4,351)$ & $4,793(7,176)$ & 0,002 \\
\hline 2 & $2,557(2,809)$ & $5,706(7,862)$ & 0,000 \\
\hline 3 & $2,534(3,391)$ & $3,696(6,333)$ & 0,000 \\
\hline 4 & $1,916(1,760)$ & $1,941(2,817)$ & 0,348 \\
\hline 5 & $1,975(2,519)$ & $2,596(4,919)$ & 0,574 \\
\hline 6 & $2,143(1,701)$ & $1,556(0,863)$ & 0,051 \\
\hline 7 & - & - & - \\
\hline 8 & - & - & - \\
\hline 9 & - & - & - \\
\hline
\end{tabular}

Tableau IV - Moyenne, par strate (sous-bois ; canopée) et par station (1-6), du nombre d'exemplaires d'Arthropodes collectés au battage et à l'aide de pièges jaunes.

Tirets : données insuffisantes. 


\begin{tabular}{|c|c|c|c|c|}
\hline \multirow{2}{*}{ Groupes écologiques } & \multicolumn{2}{|c|}{ Moyenne du nombre d'exemplaires } & \multirow{2}{*}{$\begin{array}{c}\text { t-test } \\
P\end{array}$} & \multirow{2}{*}{$\begin{array}{c}\text { Mann-Whitney } \\
\text { P }\end{array}$} \\
\hline & Sous-bois & Canopée & & \\
\hline Phyllophages (Phy) & $1,448(1,017)$ & $1,935(3,437)$ & 0,087 & 0,374 \\
\hline Piqueurs-suceurs (Ps) & $1,123(0,367)$ & $2,434(2,938)$ & 0,000 & 0,000 \\
\hline Mycophages (Myc) & 1,000 & 1,000 & - & 1,000 \\
\hline Prédateurs d'Insectes (Pi) & $1,800(0,438)$ & $1,196(0,453)$ & 0,864 & 0,856 \\
\hline Autres prédateurs (Ap) & $3,400(1,869)$ & $1,880(1,162)$ & 0,000 & \\
\hline Parasitoïdes (Par) & 1,000 & $1,545(0,934)$ & - & 0,141 \\
\hline Gallicoles (Gal) & 0,000 & $1,400(0,894)$ & - & 1,000 \\
\hline Xylophages (Xyl) & $1,489(0,804)$ & $1,474(0,841)$ & 0,945 & 0,871 \\
\hline Saprophages (Sap) & $1,224(0,421)$ & $1,367(0,765)$ & 0,349 & 0,740 \\
\hline Formicidae (For) & $3,259(4,771)$ & $5,585(7,359)$ & 0,083 & 0,064 \\
\hline Touristes (Tou) & 1,000 & 1,000 & - & 1,000 \\
\hline Inconnus (Inc) & 1,000 & $1,167(0,408)$ & - & 0,317 \\
\hline
\end{tabular}

Tableau V - Moyenne, par guilde et par strate, du nombre d'exemplaires d'Arthropodes collectés au battage. Tirets : données insuffisantes.

\begin{tabular}{|l|c|c|c|c|}
\hline \multirow{2}{*}{ Groupes écologiques } & \multicolumn{2}{|c|}{ Moyenne du nombre d'exemplaires } & t-test & Mann-Whitney \\
\cline { 2 - 3 } & Sous-bois & Canopée & P & P \\
\hline Phyllophages (Phy) & $2,774(2,905)$ & $5,000(4,919)$ & 0,005 & 0,022 \\
\hline Piqueurs-suceurs (Ps) & $2,2807(2,892)$ & $4,377(6,806)$ & 0,000 & 0,005 \\
\hline Mycophages (Myc) & $1,469(1,140)$ & $1,939(2,249)$ & 0,005 & 0,027 \\
\hline Prédateurs d'Insectes (Pi) & $1,156(0,424)$ & $1,405(1,231)$ & 0,219 & 0,602 \\
\hline Autres prédateurs (Ap) & $1,286(0,469)$ & $1,333(0,707)$ & 0,861 & 0,869 \\
\hline Parasitö̈des (Par) & $6,060(7,574)$ & $7,750(15,499)$ & 0,473 & 0,907 \\
\hline Gallicoles (Gal) & $6,135(5,271)$ & $2,871(2,778)$ & 0,002 & 0,001 \\
\hline Xylophages (Xyl) & $1,522(0,846)$ & $1,182(0,395)$ & 0,092 & 0,216 \\
\hline Saprophages (Sap) & $3,026(2,729)$ & $4,170(7,650)$ & 0,318 & 0,407 \\
\hline Formicidae (For) & $2,400(1,882)$ & $4,600(12,850)$ & 0,522 & 0,211 \\
\hline Touristes (Tou) & $1,188(0,445)$ & $3,357(3,498)$ & 0,000 & 0,000 \\
\hline Inconnus (Inc) & $1,455(0,820)$ & $6,193(5,340)$ & 0,000 & 0,000 \\
\hline
\end{tabular}

Tableau VI - Moyenne, par guilde et par strate, du nombre d'exemplaires d'Arthropodes collectés à l'aide de pièges jaunes.

\begin{tabular}{|c|c|c|c|c|}
\hline Méthode de collecte & Total & Nb prélèvements & Moyenne & Comparaison \\
\hline \multicolumn{5}{|c|}{ Madagascar } \\
\hline Battage & 3211 & 425 & 7,5 & $\times 1,5$ \\
\hline Pièges jaunes & 8008 & 141 & 56,8 & $\times 1,3$ \\
\hline Total & 11219 & & & $\times 1,4$ \\
\hline \multicolumn{5}{|c|}{ Gabon } \\
\hline Battage & 2469 & 363 & 6,8 & - \\
\hline Pièges jaunes & 5242 & 392 & 13,3 & - \\
\hline Total & 7711 & & & - \\
\hline
\end{tabular}

Tableau VII - Moyenne du nombre d'exemplaires d'Arthropodes collectés au battage et au piège jaune à Madagascar et au Gabon. 
4 - Parasitoïdes (Par) [Scelionidae et Platygastridae] ;

5 - Gallicoles (Gal) [Cecidomyiidae] ;

6 - Saprophages (Sap) [Blattodea et Gryllidae] ;

7 - Formicidae (For) ;

8 - Touristes (Tou) [Tabanidae].

Dans le tableau III, on peut voir en détail les guildes rencontrées dans la canopée supérieure et dans le sous-bois :

- la moyenne du nombre d'Arthropodes collectés est significativement plus élevée dans la canopée supérieure que dans le sous-bois pour les guildes Phyllophages (Phy), Piqueurs-suceurs (Ps), Touristes (Tou) et Inconnus (Inc) ;

- la moyenne du nombre de Piqueurs-suceurs (Ps) est selon les familles significativement plus élevée dans la canopée (Aphididae, Cicadellidae) ou le sous-bois (Cercopidae) ;

- la moyenne du nombre d'Arthropodes collectés est significativement plus élevée dans le sous-bois que dans la canopée supérieure pour les Autres prédateurs (Ap) et les Gallicoles (Gal) ;

- on ne trouve pas de différence significative entre les strates pour les Curculionidae, les Tettigoniidae, les guildes Mycophages (Myc), Prédateurs d'Insectes (Pi), Parasitoïdes (Par), Xylophages (Xyl), Saprophages (Sap) et Formicidae (For).

L'abondance des Arthropodes varie de façon significative entre les strates des trois premières stations d'échantillonnage, mais pas dans le reste des stations (tableau IV).

\section{REMARQUES SUR L'ENSEMBLE DES ÉCHANTILLONS COLLECTÉS}

\section{Battage}

En utilisant le test de Friedman pour analyser la variance, on constate que l'abondance générale des Arthropodes varie de manière significative $(p<0,0001)$ en fonction de la strate. L'abondance des Arthropodes est significativement plus élevée dans la canopée supérieure que dans le sous-bois : la moyenne du nombre d'exemplaires par strate est de 3,4 pour la canopée et de 2,8 pour le sous-bois ; Mann-Whitney $U=$ $1664153,5(\mathrm{p}=0,020)$.

De la même façon, l'abondance des Arthropodes varie de manière significative ( $p=0,000)$ selon les stations d'échantillonnage. L'abondance des Arthropodes collectés dans le sous-bois de l'ensemble des stations d'échantillonnage varie significativement selon celles-ci $(p=0,003)$. De même, l'abondance des Arthropodes de la canopée supérieure $(p=0,000)$ varie de manière hautement significative si l'on compare les diverses stations d'échantillonnage entre elles. En analysant les données par guildes, nous observons que l'abondance des Arthropodes dans la canopée supérieure et dans le sous-bois varie de façon hautement significative $(p=0,000)$. De plus, d'une manière 
générale, l'abondance des Arthropodes varie de façon hautement significative ( $\mathrm{p}=$ 0,000 ) pour chacune des neuf guildes et dans chaque station d'échantillonnage.

\section{Pièges à glu}

Nous avons placé 141 pièges à glu avec lesquels nous avons capturé 8008 Arthropodes, avec une moyenne de 56,7 exemplaires par piège.

En général, l'abondance des Arthropodes collectés par cette technique varie de manière significative selon les strates (tableau VI) ( $t$-test $p=0,002)$, Mann-Whitney $U$ $=530965,5(\mathrm{p}=0,000)$.

La quantité d'Arthropodes ne varie pas de façon significative entre les différentes zones de collecte $(p=0,070)$.

En faisant une analyse par groupe écologique et par zone d'échantillonnage, nous constatons que la quantité d'Arthropodes varie de façon hautement significative ( $\mathrm{p}=$ $0,000)$.

\section{CONSIDÉRATIONS SUR L'ANALYSE DES STATIONS D'ÉCHANTILLONNAGE 3-6 ET 8}

Nous avons capturé 6362 Arthropodes dans ces cinq stations, avec une moyenne de 17 exemplaires par échantillon. Les Arthropodes de la canopée supérieure sont significativement plus abondants que ceux provenant du sous-bois ( $t$-test, $p=0,000$ ) ; MannWhitney $U=724067,5(p=0,010)$. L'abondance des Arthropodes varie de façon significative entre les canopées supérieures des diverses stations d'échantillonnage ( $p=$ 0,000 ), mais elle ne varie pas d'une manière aussi significative dans le cas des différents sous-bois explorés $(p=0,044)$. En analysant la quantité d'Arthropodes selon les neuf guildes, nous voyons qu'il y a des variations significatives entre les deux strates ( $\mathrm{p}=$ 0,000 ).

\section{Battage}

Nous avons collecté 446 échantillons de battage dans les neuf stations d'échantillonnage, dont 234 dans la canopée et 212 dans le sous-bois.

De façon générale, la quantité d'Arthropodes ne varie pas de manière significative entre les strates $(U=319115,0 ; p=0,113)$. Parmi ces échantillons, nous en avons analysé 375 de façon plus détaillée (184 de la canopée et 191 du sous-bois), prélevés dans les stations d'échantillonnage $3,4,5$ et 6 (dans la mesure où nous avions pu réaliser pour celles-ci le même effort d'échantillonnage ? par battage ? dans la canopée et dans le sous-bois). L'analyse de ces quatre stations, pour cette même technique de prélèvement, montre qu'il n'y a pas de différence significative entre les strates de ces sites pour les Arthropodes en général.

Nous avons capturé 2682 exemplaires d'Arthropodes dans ces quatre stations, avec une moyenne de 8,2 individus par prélèvement. Pour chacun de ces quatre sites de 
battage, la quantité d'Arthropodes capturés dans la canopée et le sous-bois ne présente pas de différence significative ( $t$-test, $p=0,127$ ) ; Mann-Whitney $U=216107,500(p=$ 0,688 ). En ce qui concerne la quantité d'Arthropodes prélevés, il n'y a pas de différences significatives entre les canopées explorées, ni d'ailleurs entre les sous-bois correspondants $(p=0,116),(p=0,211)$. La quantité des Arthropodes ne varie pas de façon significative entre les deux strates de toutes les stations d'échantillonnage (sous-bois : $p=0,211$; canopée : $p=0,116$ ). L'abondance des Arthropodes par lieu d'échantillonnage ne varie pas significativement non plus.

Des différences entre les strates apparaissent lorsque les Arthropodes sont classés au sein des guildes. En analysant la quantité d'Arthropodes selon les neuf guildes, nous voyons qu'il y a des variations significatives dans les deux strates $(p=0,000)$. En particulier, nous observons que les Autres prédateurs (Ap) [Araignées] sont plus abondants dans le sous-bois et que les Piqueurs-suceurs (Ps) sont plus abondants dans la canopée. Pour les autres groupes écologiques, l'abondance ne varie guère d'une strate à l'autre (tableau V).

Après application du test Mann-Whitney, on peut constater qu'il n'y a pas de différence significative entre les sites pour les guildes, sauf Phy, Ap et For.

\section{Pièges à glu}

Pour la station d'échantillonnage 3, nous avons posé 79 pièges jaunes qui ont englué 3680 Arthropodes, avec une moyenne de 46,5 exemplaires par piège.

En général, la quantité des Arthropodes capturés par ce moyen, dans cette station, varie de façon significative selon les strates. Mann-Whitney : $U=142734(p=0,001)$.

Lorsque nous analysons les données par guilde, nous pouvons voir que l'abondance des Arthropodes dans la canopée est plus élevée que dans le sous-bois pour les guildes Phy, Ps, Myc, Tou et Inc. Au contraire, la guilde Gal est plus abondante dans le sous-bois que dans la canopée. Pour les autres guildes, il n'y a pas de différences significatives entre les strates (tableau VI).

\section{DISCUSSION}

À Madagascar, nous avons collecté au battage et avec les pièges jaunes 1,4 fois plus d'Arthropodes (11 219 exemplaires) qu'au Gabon (7 711 exemplaires) (BASSET et al., 2001). Si l'on compare les résultats par méthode utilisée, nous avons pris au battage 1,5 fois plus et avec les pièges jaunes 1,3 fois plus d'exemplaires à Madagascar qu'au Gabon. Si nous affinons ces données brutes en corrigeant par le nombre de prélèvements respectifs et par le nombre et la surface collectrice des pièges jaunes employés dans les deux pays, cette tendance se confirme. Nous avons pris au Gabon 2469 Arthropodes dans 363 échantillons de battage, soit en moyenne 6,8 exemplaires par échantillon ; à Madagascar 3211 Arthropodes ont été collectés dans 425 échantillons de battage, soit 7,5 exemplaires par échantillon. Nous avons pris au Gabon 5242 Arthropodes englués sur 392 pièges jaunes, soit en moyenne 13,3 biotes par piège ; à Madagascar 8008 Arthropodes ont été englués sur 141 pièges jaunes, soit 56,8 biotes par échantillon 
(tableau VII). Cette tendance s'accentue si on tient compte de la surface de chaque piège jaune à Madagascar $\left(638,4 \mathrm{~cm}^{2}\right)$ et au Gabon $\left(725 \mathrm{~cm}^{2}\right.$, soit $\left.\times 1,1356\right)$. Si on suppose que le nombre d'échantillons collectés est linéairement proportionnel à la surface du piège jaune (ce qui est discutable), on passe à une valeur corrigée de 64,5 biotes par piège jaune à Madagascar.

Cette apparente plus grande abondance de l'entomofaune à Madagascar qu'au Gabon n'est pas confirmée par la quantité d'Insectes pris aux pièges Malaise placés en forêt des Abeilles (la Makandé) et à Masoala (pointe Tampolo) : nous avons collecté entre dix et cent fois plus d'échantillons au Gabon qu'à Madagascar, avec pourtant le même nombre de pièges Malaise placés pendant la même durée. À vrai dire, nous n'avons constaté nulle part ailleurs en forêt tropicale que des pièges Malaise puissent être aussi peu productifs ! Les pièges d'interception ont eu un excellent rendement au Gabon et un résultat quasi nul à Madagascar. Les pièges lumineux furent beaucoup plus attractifs en nombre d'espèces et en nombre d'exemplaires à la Makandé (où le drap et les alentours du piège étaient noirs d'insectes) qu'à Tampolo (où le drap restait blanc). De même, le fauchage de la végétation du sous-bois a été moins productif à Masoala qu'en forêt des Abeilles. Si la saison de collecte, moins favorable à Madagascar et idéale au Gabon, peut sans doute partiellement rendre compte de ces différences, cela n'explique pas pourquoi le battage et les pièges à glu furent par contre plus productifs à Tampolo qu'à la Makandé.

La quantité d'exemplaires collectés dans la canopée supérieure n'est pas très différente de celle du sous-bois, indépendamment des techniques d'échantillonnage utilisées (battage et pièges jaunes). Néanmoins, nous devons examiner plus en détails les facteurs qui pcuvent biaiscr nos résultats. Les pièges jaunes sont en général plus efficaces pour capturer les Arthropodes petits et/ou de faible poids (ROBINSON \& RoBINSON, 1973), les plus grands parvenant à se décoller et à s'enfuir. Par ailleurs, BASSET et al. (2001) mentionnent qu'au Gabon les pièges jaunes placés dans la canopée pouvaient être mieux vus à cause de la luminosité plus importante dans cette strate. Si l'on compare cette forêt malgache à celle étudiée au Gabon, la couverture végétale est moins dense et la forêt atteint une hauteur sensiblement inférieure, le sous-bois est donc plus lumineux. Cette différence entre les deux forêts peut affecter la quantité d'Arthropodes présents dans les deux strates, canopée et sous-bois. Il est évident que certains Insectes s'échappent au cours du battage parce qu'ils volent très bien, mais ce problème est atténué par l'heure très matinale des vols de luge : les Insectes sont encore mouillés et engourdis par la fraîcheur de la nuit. Il faut aussi reconnaître que les techniques de collecte utilisées ne permettent pas d'échantillonner tous les Arthropodes présents sur les sites. Avec les techniques utilisées, nous pouvons bien mieux échantillonner les Insectes adultes ailés et mobiles et moins bien les larves. D'autres facteurs, comme les fortes rafales de vent qui agitent la canopée supérieure, peuvent influencer la capture des Insectes volants, surtout si on compare ces perturbations parfois violentes à la tranquillité constante de l'atmosphère dans le sous-bois, particulièrement dans le cas des Insectes moins actifs (SUTTON \& HuDson, 1980).

Il faut également souligner que la répartition hétérogène des échantillons collectés dans les différentes stations d'échantillonnage doit être prise en compte pour une bonne interprétation des résultats. Les ressources disponibles dans les différentes stations d'échantillonnage, que les Insectes utilisent pour vivre, sont un paramètre dont 
nous ne pouvons pas faire abstraction dans notre analyse. Divers problèmes logistiques nous ont empêchés de réaliser un échantillonnage homogène pour toutes les stations, ce qui a eu des répercussions sur la comparaison des résultats de façon plus généralisée.

On trouve la plus grande abondance et la plus grande diversité de phytophages dans la canopée médiane ou supérieure (BASSET et al., 1992, 2001 ; BARRIOS, 2003). Cela peut s'expliquer en partie par le fait qu'il existe dans la canopée un vaste éventail d'habitats que l'on ne trouve pas dans le sous-bois. Par exemple, la plus grande biomasse et la plus grande diversité de structures de lianes se trouve dans la canopée médiane ou supérieure (HEGARTY \& CABALlÉ, 1991) et l'on trouve associée à celle-ci une grande diversité d'Insectes phytophages (STORK, 1987 ; ØEDEGAARD, 2000). L'indice de surface foliaire, plus faible dans le sous-bois que dans la canopée supérieure, n'affecte pas seulement les ressources alimentaires en elles-mêmes, mais aussi suppose un moindre choix de micro-abris pour se protéger des prédateurs.

\section{CONCLUSIONS}

Un facteur important ayant affecté le modèle de stratification verticale est la hauteur de la forêt, moins élevée dans les stations étudiées dans le parc de Masoala qu'à la Makandé, au Gabon. La luminosité du sous-bois est relativement élevée, ce qui d'une manière directe ou indirecte a eu des répercussions sur les résultats de notre étude. Les forêts tropicales caractérisées par une stratification marquée ont une hauteur égale ou supérieure à 35 mètres et une forte densité foliaire, ce qui entraîne une forte stratification verticale de l'entomofaune.

Il faut souligner :

a) que les Arthropodes collectés ne sont plus abondants dans la canopée supérieure que dans le sous-bois que dans la moitié des stations étudiées ;

b) que l'analyse des données par famille et par guilde montre que certains de ces ensembles ont nettement une strate préférentielle, soit la canopée, soit le sous-bois, ce qui est directement lié aux ressources alimentaires que chaque groupe exploite ;

c) que l'assignation à une guilde semble donc être un instrument pertinent pour interpréter la réalité biologique de la forêt tropicale, en situant les Arthropodes dans leurs niches écologiques;

d) que les Insectes phytophages appartenant au groupe des Piqueurs-suceurs présentent une grande variabilité en termes de stratification verticale et de répartition horizontale.

Pour les Arthropodes peuplant la forêt tropicale humide, le modèle de stratification verticale observé en Afrique se retrouve donc confirmé dans ses grandes lignes à Masoala (BASSET et al., 1992, 2001, 2003).

Remerciements. - Nous remercions chaleureusement toutes celles et tous ceux qui ont permis que cette mission se déroule dans les meilleures conditions. Les représentants des autorités malgaches: Michel Ranarivelo et Robert Rajaonarison ; l'équipe d'Océan Vert : Francis Hallé, Dany Cleyet-Marrel, Jean Drouault, Gilles Ebersolt et Laurent Pyot ; l'équipe de Pro-Natura International : Karine Genser et Olivier Pascal ; 
les grimpeurs et bricoleurs de génie : Thierry Aubert (qui a tiré HPA d'une situation périlleuse avec la bulle), Noui Beben, François Klene, Nicolas Lochu, Frédéric Mathias et Jean-Yves Serein; les porteurs : Georges et $\mathrm{Mi}$; le médecin de l'expédition : Guilhem Peremarty ; l'équipe de cinéma : Antoine de Maximy, Jean-Baptiste Benoît et Luc Marescot ; nos collègues présents sur le terrain : Lanto Andriamampianina, Eric Faure, Thomas Haevermans, Harold Heatwole, Jean-Noël Labat, David Lees, Odile Poncy, Casimir Rafamantanantsoa, Maminirina Randrianandrasana, Emilienne Razafimahatratra, Andrianantenaina Razafindrakotomamonjy et Sybille Unsicker; les collègues qui n'ont pas pu venir: Yves Basset et Jean-Marc Elouard ; notre traductri$c e$ : Nathalie Cantero; nos mécènes : Gérard Eyriès et Stéphanie Gorre-Matillon de Bayer-Agro (financement du voyage d'HPA et d'HB) ; le Parco Regionale del fiume Po, Sezione Torinese (financement partiel du voyage de GC); le CIRAD-CP, Programme Cocotier (financement du voyage et du séjour de LO); le photographe : Hirochika Setsumasa.

\section{RÉFÉRENCES BIBLIOGRAPHIQUES}

Aberlenc H.-P., Andriamampianina L., Faure E., Lees D. C., Minet J., Ollivier L., Rafamantanantsoa C., Randrianandrasana M. et Razafindrakotomamonjy A., 2007 - Le Radeau des Cimes au Parc national de Masoala (Madagascar). Première partie : éléments pour un inventaire des Lépidoptères. Bulletin mensuel de la Société linnéenne de Lyon, 76 (6) : 141-154.

BARRIOS H., 2003 - Insect herbivores feeding on conspecific seedlings and trees: 282-290. In : BASSET Y., Novotny V., Miller S. E. et KITching R. L. (éd.) : Arthropods of tropical forest. Spatio-temporal dynamics and resource use in the canopy. Cambridge University Press, $\mathrm{xvi}+474 \mathrm{p}$.

BASSET Y., 1992 - Synecology and aggregation patterns of arboreal arthropods associated with an overstorey rainforest tree. Journal of Tropical Ecology, 8 : 317-327.

Basset Y., Delvare G. et Aberlenc H.-P., 1992 - Abundance and stratification of foliage arthopods in a lowland rain forest of Cameroon. Ecological Entomology, $17: 310-318$.

Basset Y. et Novotny V., 1999 - Species richness of insect herbivores on Ficus in Papua New Guinea. Biological Journal of the Linnean Society, 67: 477-499.

Basset Y., Aberlenc H.-P., Barrios H., Curletti G., Béranger J.-M., Vesco J.-P., Causse P., Haug A., Hennion A.-S., Lesobre L., Marques F. et O'Meara R., 2001 - Stratification and diel activity of arthropods in a lowland rainforest in Gabon. Biological Journal of the Linnean Society, 72 : 585-607.

Basset Y., Samuelson G. A., Allison A. et Miller S. E., 1996 - How many species of host-specific insect feed on a species of tropical tree ? Biological Journal of the Linnean Society, 59 : 201-216.

Basset Y., Hammond P. M., Barrios H., Holloway J. D. et Miller S. E., 2003 - Vertical stratification of arthropod assemblages : 17-27. In : BASSET Y., Novotny V., Miller S. E. et Kitching R. L. (éd.) : Arthropods of tropical forests. Spatio-temporal dynamics and resource use in the canopy. Cambridge University Press, xvi +474 p.

Bell A. D., Bell A. et Dines T. D., 1999 - Branch construction and bud defence status at the canopy surface of a West African rainforest. Biological Journal of the Linnean Society, 66 : $481-499$.

Blanc P., 1990 - Bioclimatologie comparée de la canopée et du sous-bois : 42-43. In : HaLlÉ F. et BlANC P. (éd.) : Biologie d'une canopée de forêt équatoriale. Rapport de mission : Radeau des Cimes Octobre-Novembre 1989, Petit Saut - Guyane Française. Montpellier II et CNRS-Paris VI, $231 \mathrm{p}$.

Cleyet-Marrel D., 1999 - La bulle des cimes : 20-21. In : Hallé F. (éd.) : Biologie d'une canopée de forêt équatoriale - IV. Rapport de mission : Radeau des Cimes Janvier-Mars 1999, La Makandé, Gabon. Paris, Pro-Natura International \& Opération Canopée, 168 p.

Col.wF.... R. K., 1997 - Biota: the Biodiversity Database Manager [logiciel]. Sunderland, MA, Sinauer Associates. 
Ebersolt G., 1990 - Opération "Radeau des Cimes", Mission 1989 - Guyane. Bilan technique partie radeau : 15-26. In : HALlÉ F. et BlANC P. (éd.) : Biologie d'une canopée de forêt équatoriale. Rapport de mission : Radeau des Cimes Octobre-Novembre 1989, Guyane Française. Montpellier II et CNRS-Paris VI, $231 \mathrm{p}$.

Ebersolt G., 1999 - Icos et Radeau. La bulle des cimes : 22-24. In : HAllÉ F. (éd.) : Biologie d'une canopée de foret équatoriale - IV. Rapport de mission : Radeau des Cimes Janvier-Mars 1999, La Makandé, Gabon. Paris, Pro-Natura International \& Opération Canopée, 168 p.

ERwiN T. L., 1991 - How many species are there: revisited. Conservation Biology, 5 : 330-333.

ERWIN T. L., 1995 - Measuring arthropod biodiversity in the tropical forest canopy: 109-126. In : I owman M. D. et Nadkarni N. M. (éd.) : Forest canopies. San Diego, Academic Press.

FOWLER S. V., 1985 - Difference in insect species richness and faunal composition of birch seedlings, saplings and trees: the importance of plant architecture. Ecological Entomology, $10: 159-169$.

HALLÉ F., 1998 - Distribution verticale des métabolites secondaires en forêt équatoriale - une hypothèse. In : HALLÉ F. (éd.) : Biologie d'une canopée de forêt équatoriale - III. Rapport de la mission d'exploration scientifique de la canopée de Guyane, Octobre-Décembre 1996. Paris, Pro-Natura International \& Opération Canopée, p. 129-138.

Hegarty E. E. et CABALLÉ G., 1991 - Distribution and abundance of vines in forest communities : 313335. In : Putz F. E. et Mooney H. A. (éd.): The Biology of Vines. Cambridge University Press.

JANZEN D. H., 1988 - Ecological characterization of a Costa Rican dry forest caterpillar fauna. Biotropica, $20: 120-135$.

Martinez M. et GaUVRit B., 1997 - Combien y a-t-il d'espèces d'Insectes en France ? Bulletin mensuel de la Société entomologique de France, 102 (4) : 319-332.

MoRAN C. V. et Southwood T. R. E., 1982 - The guild composition of arthropod communities in trees. Journal of Animal Ecology, 51 : 289-306.

ØDEGAARD F., 2000 - The relative importance of trees versus lianas as hosts for phytophagous beetles (Coleoptera) in tropical forests. Journal of Biogeography, 27 : 283-296.

PARKER G. G., 1995 - Structure and microclimate of forest canopies: 431-455. In : LowMAN M. D. et Nadkarni N. M. (éd.) : Forest Canopies. San Diego, Academic Press.

Paulian R., 1961 - La zoogéographie de Madagascar et des îles voisines. Paris, Coll. Faune de Madagascar, $13: 1-485$.

Robinson M. H. et RoBinson B., 1973 - Ecology and behavior of the giant wood spider Nephila maculata (Fabricius) in New Guinea. Smithsonian Contributions to Zoology, 149 : iv + 1-76.

RouBik D. W., 1993 - Tropical pollinators in the canopy and understorey: field data and theory for stratum "preferences". Journal of Insect Behavior, $6: 659-673$.

Schowalter T. D., HaRgrove, W. W. et Crossley D. A. Jr., 1986 - Herbivory in forested ecosystems. Annual Review of Entomology, 31 : 177-196.

SHELLY T. E., 1985 - Ecological comparisons of robber fly species (Diptera : Asilidae) coexisting in a neotropical forest. Oecologia, $67: 57-70$.

STORK N. E. 1987 - Arthropod faunal similarity of Bornean rain forest trees. Ecological Entomology, $12: 219-226$.

SutTon S. L. et Hudson P. J., 1980 - The vertical distribution of small flying insects in the lowland rain forest Zaire. Zoological Journal of the Linnean Society, 68 : 111-123.

Vitali F., 2006 - Contribution à la connaissance des Nethinius malgaches (Coleoptera Disteniidae). L'Entomologiste, 62 (5-6) : 175-178.

WILSON E. O., 1988 - The current state of biological diversity: 3-18. In : WILSON E. O. (éd.): Biodiversity. Washington, National Academy Press.

WILSON E. O., 1989 - Threats to biodiversity. Scientific American : 109-116.

WILSON E. O., 1992 - The diversity of life. Cambridge, Harvard University Press, vii+ 424 p.

WRIGHT S. J. et Colley M. (éd.), 1994 - Accessing the canopy. Assessment of biological diversity and microclimate of the tropical forest canopy: Phase I. Nairobi, United Nations Environment Programme. 